\title{
Differential outcomes of type A dissection with malperfusion according to affected organ system
}

\author{
Joshua C. Grimm ${ }^{1}$, J. Trent Magruder ${ }^{1}$, Todd C. Crawford ${ }^{1}$, Christopher M. Sciortino ${ }^{1}$, Kenton J. Zehr ${ }^{1}$, \\ Kaushik Mandal $^{1}$, John V. Conte ${ }^{1}$, Duke E. Cameron ${ }^{1}$, James H. Black ${ }^{2}$, Joel E. Price ${ }^{3}$ \\ ${ }^{1}$ The Division of Cardiac Surgery, ${ }^{2}$ Division of Vascular Surgery and Endovascular Therapy, The Johns Hopkins Hospital, Baltimore, MD, USA; \\ ${ }^{3}$ Division of Cardiac Surgery, University of British Columbia, Vancouver, Canada \\ Correspondence to: Joel E. Price, MD, MPH. 494-1081 Burrard Street, Vancouver, BC, Canada. Email: joel.price@vch.ca.
}

Background: The management of malperfusion in patients with acute Stanford type A aortic dissection is controversial. We sought to determine the rate of resolution of malperfusion following primary repair of the dissection and to identify anatomic sites of malperfusion that may require additional management.

Methods: We reviewed the hospital records of patients who presented to our institution with acute type A aortic dissection. Patient demographics, operative details and post-operative course were retrospectively extracted from our institutional electronic database. Depending upon the anatomic site, malperfusion was identified by a combination of radiographic and clinical definitions. Data were analyzed using standard univariable and multivariable methods.

Results: Between 1997-2013, 101 patients underwent repair of an acute type A dissection. Thirty-day mortality was $14.9 \%$ (15/101); there were five intraoperative deaths. There was no difference in 30-day mortality between patients with or without malperfusion $(15.4 \%$ vs. $14.7 \%, \mathrm{P}=0.93)$. Twenty-five patients $(24.7 \%)$, who survived surgery, presented with 31 sites of malperfusion. Anatomic sites included extremities [14], renal [10], cerebral [5] and intestinal [2]. Of these 31 sites, malperfusion resolved in 18 (58.1\%) with primary aortic repair. Renal malperfusion resolved radiographically in $80.0 \%$, with no difference in the incidence of insufficiency (44.0\% vs. $35.2 \%$; $\mathrm{P}=0.44)$ or dialysis $(20.0 \%$ vs. $15.5 \% ; \mathrm{P}=0.61)$ between malperfusion and non-malperfusion patients. Extremity malperfusion resolved postoperatively in six out of 14 patients. Of the remaining eight, concomitant revascularization was performed in four, one had an amputation and three required postoperative interventions. Advanced patient age (OR: 1.06, 95\% CI: 1.01$1.12, \mathrm{P}=0.02)$ was an independent predictor of 30-day mortality, while preoperative malperfusion was not (OR: 0.77, 95\% CI: 0.18-3.31, P=0.73).

Conclusions: Malperfusion complicating acute type A dissection can be managed in many patients by aortic replacement alone with low overall mortality. Most cases of renal and cerebral malperfusion resolved following aortic surgery. Revascularization was frequently necessary in patients with extremity malperfusion. Patients presenting with intestinal ischemia had very poor outcomes. A patient-specific approach is recommended in such complex patients.

Keywords: Aortic dissection; malperfusion; short-term survival

Submitted Jan 25, 2016. Accepted for publication Feb 01, 2016.

doi: 10.21037/acs.2016.03.11

View this article at: http://dx.doi.org/10.21037/acs.2016.03.11 


\section{Introduction}

Though its incidence is low (2.9 per 100,000), acute type A aortic dissection is associated with considerable morbidity and mortality $(1,2)$. End-organ malperfusion that results from propagation of the dissection flap or subsequent arterial thrombosis complicates $15-40 \%$ of all cases $(2,3)$. The repair strategy in complex dissections involving the cerebral, visceral, extremity or other side-branch arteries remains controversial.

In the traditional "aortic-first" approach, reconstitution of true lumen flow is accomplished with an ascending aorta or hemiarch repair. Peripheral revascularization is only pursued if there is evidence of ongoing end-organ ischemia after the primary surgery. Opponents of this technique argue in favor of initial percutaneous or surgical revascularization to address end-organ injury in the hope of optimizing patient selection prior to definitive aortic repair (4-6). The aortic surgery is performed after a variable period of end-organ stabilization. The inherent danger of this staged approach is interim aortic rupture with catastrophic hemorrhage despite aggressive medical management.

Given the uncertainty of optimal management strategy of these challenging patients, we examined our institutional experience with acute proximal dissections presenting with end-organ malperfusion, in order to gain a better understanding of end-organ reperfusion and recovery following prompt aortic surgery and to identify anatomic sites which might require more frequent additional interventions.

\section{Methods}

\section{Patient selection}

We searched our institutional database for all adult patients ( $\geq 18$ years) presenting with an acute type A aortic dissection from January 1, 1997 to December 31, 2013. The definition of an 'acute' dissection was based on a duration of symptoms of less than 14 days prior to presentation (7). Patients with a sub-acute or chronic dissection and those that did not undergo surgery were excluded from further analysis.

Operations were performed by a number of surgeons over the study period. As a result, there was some variation in the surgical technique. However, circulatory arrest with varying degrees of hypothermia was utilized in all patients. In all cases, the ascending aorta was replaced with a Dacron tube graft. The decision to proceed with a tube graft repair or a more extensive hemiarch or total arch replacement and/or aortic root replacement was based on intraoperative findings and surgeon judgment. For cases presenting with end-organ ischemia, all but four cases underwent a primary proximal aortic operation with the intention of relieving the malperfusion syndrome. In the four cases of limb ischemia requiring concomitant vascular procedures, the following were performed: femoral-femoral bypass in two cases, an aortobifemoral bypass after failed transarterial measures and a left iliofemoral bypass with left common femoral artery endarterectomy.

\section{Definition of malperfusion and its resolution}

The definition of malperfusion employed in this study varied based upon anatomic site. Radiographic evidence of visceral (renal, celiac, or superior mesenteric artery), coronary, and cerebral malperfusion was required with a contrast enhanced computed tomography scan. Physical exam findings consistent with limb ischemia (loss of pulse) were adequate for an extremity definition of malperfusion. Confirmation of the clinical findings was obtained from a combination of admission and operative notes.

The classification of 'resolved' malperfusion varied according to the affected end-organ and can be summarized by the following:

(I) Renal: return to baseline serum creatinine without long-term hemodialysis;

(II) Intestinal: resolution of lactic acidemia without clinical signs of ongoing intestinal ischemia (gastrointestinal hemorrhage);

(III) Extremity: restoration of adequate perfusion to the extremity as evidenced by physical exam, such as return of pulses and clinical evidence of limb viability;

(IV) Cerebral: absence of permanent defects on neurologic examination.

\section{Statistical analysis}

Standard descriptive analysis, including baseline and operative factors, was performed. Thirty-day survival was reported for the entire cohort and by organ system for malperfusion patients. For patients with multiple sites of malperfusion, each organ system was treated as a separate instance for the reperfusion analysis. The incidence of malperfusion resolution following aortic repair was determined for each organ system. The need for renal replacement therapy was also analyzed in those patients presenting with renal malperfusion. Non-parametric, 


\begin{tabular}{|c|c|c|c|}
\hline Variable & Malperfusion $(n=26)$ & No malperfusion $(n=75)$ & $P$ value \\
\hline Age (years) & $57.5[46-63]$ & $59[45-67]$ & 0.72 \\
\hline Male gender & $76.9 \%$ [20] & $62.7 \%[47]$ & 0.19 \\
\hline Race & & & 0.58 \\
\hline White & $50.0 \%[13]$ & $61.3 \%[46]$ & \\
\hline Black & $46.2 \%[12]$ & $34.7 \%[26]$ & \\
\hline Other & $3.9 \%[1]$ & $4.0 \%[3]$ & \\
\hline Tobacco use & $11.5 \%[3]$ & $13.3 \%[10]$ & 0.23 \\
\hline Hypertension & $65.4 \%[17]$ & $66.7 \%[50]$ & 0.23 \\
\hline Diabetes & $7.7 \%[2]$ & $8.0 \%[6]$ & 0.23 \\
\hline NYHA & & & 0.24 \\
\hline Class I & $11.5 \%[3]$ & $2.7 \%[2]$ & \\
\hline Class II & $11.5 \%[3]$ & $10.7 \%[8]$ & \\
\hline Class III & $19.2 \%[5]$ & $34.7 \%[26]$ & \\
\hline Class IV & $38.5 \%[10]$ & $28.0 \%[21]$ & \\
\hline Unknown & $19.2 \%[5]$ & $24.0 \%[18]$ & \\
\hline Dialysis dependence & $7.7 \%[2]$ & $4.0 \%[3]$ & 0.04 \\
\hline Cerebrovascular disease & $8.0 \%$ [2] & $8.5 \%[6]$ & 0.80 \\
\hline Previous heart surgery & $3.9 \%[1]$ & $13.3 \%[10]$ & 0.03 \\
\hline
\end{tabular}

continuous data were compared with the Wilcoxon signedrank test and are reported as median [interquartile range], whereas categorical data were compared with Fischer's exact test and chi-squared analysis (where appropriate) and are reported as \%, (n). Statistical significance was established at a $\mathrm{P}$ value less than 0.05 . StataSE 13.0 was utilized for analysis (StataCorp, College Station, Texas, USA). Multivariable analysis of the entire cohort was performed to determine the independent influence of malperfusion on 30-day survival. Covariates with a $\mathrm{P}$ value of less than 0.20 on exploratory univariate analysis were then manually included in a multivariable, logistic regression model. The Akaike Information Criterion and likelihood ratio test were referenced to compare model strengths. In addition to malperfusion, the factors included in the final model were age, hypertension and preoperative dialysis dependence.

\section{Results}

A total of 101 patients underwent repair of an acute type A aortic dissection during the study period. Thirty-day mortality was $14.9 \%(15 / 101)$. Five patients (malperfusion present in one) died intraoperatively and were excluded from the outcome analysis for resolution of malperfusion. Of the remaining 96 patients, 25 (26.0\%) presented with at least one organ system of malperfusion. Thirty-day mortality was similar between patients with and without malperfusion [15.4\% (4/26) vs. $14.7 \%$ (11/75), $\mathrm{P}=0.93$ ].

In regards to baseline demographics in those with malperfusion, the median age was 57.5 [46-63] years, $20(76.9 \%)$ were male, and the majority were Caucasian ( $\mathrm{n}=13,50.0 \%)$. Hypertension, diabetes, and NYHA Class IV heart failure were present in 17 (65.4\%), 2 (7.7\%), and $10(38.5 \%)$ of malperfused patients, respectively (Table 1$)$. The most common repair strategies were replacement of the ascending aorta with a tube graft in $11(44.0 \%)$ and hemiarch replacement in eight $(32.0 \%)$. An aortic root operation was necessary in three additional patients $(12 \%)$ and three others required a complete arch replacement (12\%). The median durations of circulatory arrest, cross clamp, and cardiopulmonary bypass were 23 [14-33], 93 [67.5-112], and 182 [164-208] minutes, respectively (Table 2).

Thirty-one anatomic sites of malperfusion were identified in 25 patients who survived their aortic operation. The sites included arm/leg in 14, renal in 10, cerebral in five and intestinal in 2. Resolution of ischemia was reported 


\begin{tabular}{llll}
\multicolumn{2}{l}{ Table 2 Intraoperative characteristics in patients with and without malperfusion } & & \\
\hline Operative variable & Malperfusion $(\mathrm{n}=26)$ & No malperfusion $(\mathrm{n}=75)$ & $\mathrm{P}$ value \\
\hline Extent of repair & & & 0.13 \\
Tube graft only & $42.3 \%[11]$ & $57.3 \%[43]$ & \\
Hemiarch & $30.8 \%[8]$ & $13.3 \%[10]$ \\
Arch & $11.5 \%[3]$ & $18.7 \%[14]$ & \\
Aortic root procedure & $11.5 \%[3]$ & $5.3 \%[4]$ & 0.25 \\
Cardiopulmonary bypass time (minutes) & $173[141-209]$ & $182[164-208]$ & 0.72 \\
Cross clamp time (minutes) & $88[60-122]$ & $93[67.5-112]$ & 0.14 \\
\hline Circulatory arrest time (minutes) & $19[12-24.5]$ & $23[14-33]$ & \\
\hline
\end{tabular}

\begin{tabular}{|c|c|c|}
\hline Anatomic site & Resolution & 30-day mortality \\
\hline Renal & $80 \%(8 / 10)$ & $10 \%(1 / 10)$ \\
\hline Intestinal & $0 \%(0 / 2)$ & $100 \%(2 / 2)$ \\
\hline Extremity & $43 \%(6 * / 14)$ & $7 \%(1 / 14)$ \\
\hline Cerebral & $80 \%(4 / 5)$ & $20 \%(1 / 5)$ \\
\hline \multicolumn{3}{|c|}{$\begin{array}{l}\text { *, seven of these patients required concomitant or subsequen } \\
\text { lower extremity revascularization. One additional patient tha } \\
\text { did not undergo revascularization required a below the knee } \\
\text { amputation. }\end{array}$} \\
\hline
\end{tabular}

in $18(57.1 \%)$ with resection of the primary tear alone. The best outcomes were noted in patients presenting with renal malperfusion $(n=10)$, with $80.0 \%$ having resolved by the time of discharge. The incidence of progression to renal insufficiency ( $44.0 \%$ vs. $35.2 \% ; \mathrm{P}=0.44$ ) or failure requiring renal replacement $(20.0 \%$ vs. $15.5 \%, \mathrm{P}=0.60)$ was similar in those with and without malperfusion. Of note, none of these patients required any interventional procedures in the postoperative period to ameliorate their renal malperfusion. Only one patient died within 30 days and they had a complicated postoperative course due largely to intestinal ischemia. This patient underwent numerous operations including a left hemicolectomy and multiple washouts. In patients presenting with extremity malperfusion (12 upper extremity and 2 lower extremity), revascularization was required in $50.0 \%(7 / 14)$. One additional patient that did not undergo concomitant or subsequent revascularization required a lower extremity amputation. Only one of these patients experienced a 30-day mortality and they had an extensive dissection involving both the SMA and at-least one renal artery. With regards to cerebral malperfusion, there was one intraoperative death in this cohort, and one patient experienced a postoperative central nervous system event. None of the patients presenting with intestinal pathology experienced resolution of their malperfusion and both expired in the postoperative period (Table 3).

Patients with malperfusion had longer hospital lengths of stay $\{20[11-36]$ vs. 12 [8-18] days, $\mathrm{P}=0.006\}$, as well as more reoperations for postoperative hemorrhage $(24.0 \% \mathrm{vs}$. $5.6 \%, \mathrm{P}=0.01)$ and a greater number of postoperative CNS events $(44.0 \%$ vs. $18.3 \%, \mathrm{P}=0.01)$.

On univariate analysis, malperfusion was not predictive of 30-day mortality (OR: 1.05, 95\% CI: 0.31-3.67, P=0.93). This lack of a relationship persisted after adjusting for high-risk factors (age, preoperative hypertension and preoperative dialysis dependence) in multivariable, logistic regression (OR: 0.77, 95\% CI: 0.18-3.31, $\mathrm{P}=0.73$ ).

\section{Discussion}

We present a single institution series of an "aortic-first" approach in the initial management of acute proximal type A aortic dissections complicated by malperfusion. Our results indicate that prompt restoration of true lumen flow can obviate the need for subsequent revascularization procedures in nearly $60 \%$ of patients presenting with malperfusion. More specifically, patients with presenting with renal and cerebral malperfusion do well with aortic repair alone. The rate of recovery is most rapid in patients with renal malperfusion, most likely due to the kidney's ability to tolerate prolonged periods of altered perfusion. Concomitant or post-operative revascularization is frequently necessary for limb salvage. Patients presenting with intestinal ischemia have a very poor prognosis, in our experience.

Ultimately, the most important consideration in regards 
to the optimal surgical strategy is the expected outcomes associated with each anatomic site of malperfusion. While it would be easier to universally advocate for either an aorticor peripherally-centric paradigm in all of these patients, it is evident that there is a variation with respect to the impact of ischemia, depending upon the affected organ system, and a patient-specific approach is required.

It is well established that cases of acute dissection presenting with end-organ malperfusion are associated with increased rates of morbidity and mortality $(8,9)$. The failure to demonstrate this in our series is almost certainly a product of a small sample size. Comparisons of outcomes between the malperfusion and non-malperfusion groups were underpowered and not the purpose of this study. Rather, we intended to examine our experience of patients presenting with end-organ ischemia and to examine the variable outcomes, stratified by which organ system was malperfused, with an approach involving repair of the ascending aorta as a primary intervention.

Several series have similarly showed that renal malperfusion is often associated with favorable outcomes following repair of a type A dissection. In a study of 221 patients with type A dissections, Geirsson identified nine with dissection flaps extending into the renal arteries. Only two of these patients experienced renal insufficiency postoperatively, but both recovered without the need for dialysis following discharge (3). It is important to differentiate, however, between cases of isolated renal malperfusion and those occurring in the context of associated mesenteric or limb malperfusion. In our experience, the outcomes in the latter context are not as encouraging.

The presence of mesenteric malperfusion has been identified as a predictor of a poor prognosis in several studies (9-12). In one of the largest series, Di Eusanio et al. reviewed the International Registry of Acute Aortic Dissection, a database compiled from 18 contributing centers and comprised of 1,809 consecutive patients presenting with an acute, type A dissection, to better understand the influence of mesenteric malperfusion on outcomes. While only present in $3.8 \%$ of the patients, intestinal ischemia was associated with considerably higher in-hospital mortality $(63.2 \%$ vs. $23.8 \%, \mathrm{P}<0.001)(10)$. These patients were commonly afflicted by multiple sites of malperfusion and were routinely managed nonoperatively (10). The in-hospital mortality was largely driven by the cohort of patients managed medically (95.2\%) and was significantly lower in those deemed operative candidates (41.7\%) (10). Similarly dismal survivals for this subset were published in smaller series by Pacini (13) and Yagdi et al. (9). Our own experience, although very small, confirms the very poor outcomes in these patients. Since mesenteric ischemia remains so devastating, perhaps a more aggressive approach involving the performance of a laparoscopy after type A repair would be of use. In such a situation, segmentally damaged bowel could be addressed before general hemodynamic compromise ensues (14).

When proximal dissections extend into the cerebral vessels, there is little evidence to support carotid repair in the absence of ongoing symptoms. Charlton-Ouw et al. reported on 43 patients with common carotid artery involvement from propagation of a proximal aortic dissection and demonstrated that 5-year stroke-free survival was similar when compared to a cohort of patients with no appreciable dissection on carotid imaging $(69.7 \%$ and 73.6\%, $\mathrm{P}=0.82)$ (15). Zieliński et al. monitored 15 patients diagnosed with cerebral malperfusion with regular carotid duplexes and reported no disease progression on followup (16). Another study by Tsukube et al. investigating the role of immediate aortic repair in patients presenting comatose confirmed that neurologic function could be recovered if surgical intervention was prioritized (17). While we did not obtain routine radiographic imaging in all patients to determine the incidence of occult CNS injury, only one patient presenting with cerebral malperfusion experienced ongoing clinical manifestations postoperatively.

The management of patients with extremity malperfusion can be challenging and can, as our series illustrates, demand simultaneous or subsequent interventions to ensure adequate luminal flow. It is important to note that 10 of the 14 patients in our series were transferred from outside hospitals. It can be assumed, therefore, that the extremity was exposed to a protracted period of ischemia prior to surgical intervention. Furthermore, it is unclear how many of these patients would have been adequately perfused following aortic repair alone. The decision to pursue concomitant lower extremity interventions was based on intraoperative assessment, as definitive criteria in this setting do not exist. In another large study by Charlton-Ouw et al., lower extremity malperfusion was diagnosed in $15 \%$ of patients; of these, $22 \%$ required additional procedures to restore distal pulsation (18), which is slightly lower than the $50 \%$ reported in our series. Furthermore, in a study by Bossone et al., a preoperative pulse deficit was associated with adverse in-hospital events, including perioperative mortality (8). 


\section{Limitations}

Our series has several limitations worth highlighting. This is a single center experience. The limited sample size prohibited the construction of robust risk-adjusted models. Additionally, while a majority of the repairs were performed by a small cohort of surgeons, advances in preoperative imaging and patient optimization, as well as changes in the operative technique over the study period, may have influenced outcomes. There is no convention with regards to the peri-, intra-, and postoperative care of patients with type A dissections with malperfusion. Subsequently, surgeon-specific preferences could have attributed to significant differences in morbidity and mortality.

\section{Conclusions}

Patients presenting with acute, type A aortic dissections complicated by malperfusion can be challenging to manage in the perioperative period. Our series demonstrates that outcomes comparable to those in patients without malperfusion can be achieved with prompt aortic repair in certain subsets of these patients. Those with renal and cerebral malperfusion do well with aortic repair alone. Patients presenting with intestinal ischemia have very poor outcomes. Concomitant or post-operative revascularization is frequently necessary for limb salvage in patients with extremity malperfusion. Therefore, a patient-specific approach is recommended in these complex patients.

\section{Acknowledgements}

None.

\section{Footnote}

Conflicts of Interest: The authors have no conflicts of interest to declare.

\section{References}

1. Mészáros I, Mórocz J, Szlávi J, et al. Epidemiology and clinicopathology of aortic dissection. Chest 2000;117:1271-8.

2. Trimarchi S, Nienaber CA, Rampoldi V, et al. Contemporary results of surgery in acute type A aortic dissection: The International Registry of Acute Aortic Dissection experience. J Thorac Cardiovasc Surg
2005;129:112-22.

3. Geirsson A, Szeto WY, Pochettino A, et al. Significance of malperfusion syndromes prior to contemporary surgical repair for acute type A dissection: outcomes and need for additional revascularizations. Eur J Cardiothorac Surg 2007;32:255-62.

4. Suliman A, Dialynas M, Ashrafian H, et al. Acute Complex Type A Dissection associated with peripheral malperfusion syndrome treated with a staged approach guided by lactate levels. J Cardiothorac Surg 2010;5:4.

5. Deeb GM, Williams DM, Bolling SF, et al. Surgical delay for acute type A dissection with malperfusion. Ann Thorac Surg 1997;64:1669-75; discussion 1675-7.

6. Patel HJ, Williams DM, Dasika NL, et al. Operative delay for peripheral malperfusion syndrome in acute type A aortic dissection: a long-term analysis. J Thorac Cardiovasc Surg 2008;135:1288-95; discussion 1295-6.

7. Hiratzka LF, Bakris GL, Beckman JA, et al. 2010 ACCF/AHA/AATS/ACR/ASA/SCA/SCAI/SIR/STS/ SVM guidelines for the diagnosis and management of patients with Thoracic Aortic Disease: a report of the American College of Cardiology Foundation/American Heart Association Task Force on Practice Guidelines, American Association for Thoracic Surgery, American College of Radiology, American Stroke Association, Society of Cardiovascular Anesthesiologists, Society for Cardiovascular Angiography and Interventions, Society of Interventional Radiology, Society of Thoracic Surgeons, and Society for Vascular Medicine. Circulation 2010;121:e266-369.

8. Bossone E, Rampoldi V, Nienaber CA, et al. Usefulness of pulse deficit to predict in-hospital complications and mortality in patients with acute type A aortic dissection. Am J Cardiol 2002;89:851-5.

9. Yagdi T, Atay Y, Engin C, et al. Impact of organ malperfusion on mortality and morbidity in acute type A aortic dissections. J Card Surg 2006;21:363-9.

10. Di Eusanio M, Trimarchi S, Patel HJ, et al. Clinical presentation, management, and short-term outcome of patients with type A acute dissection complicated by mesenteric malperfusion: observations from the International Registry of Acute Aortic Dissection. J Thorac Cardiovasc Surg 2013;145:385-90.

11. Perera NK, Galvin SD, Seevanayagam S, et al. Optimal management of acute type A aortic dissection with mesenteric malperfusion. Interact Cardiovasc Thorac Surg 2014;19:290-4.

12. Girdauskas E, Kuntze T, Borger MA, et al. Surgical risk of 
preoperative malperfusion in acute type A aortic dissection. J Thorac Cardiovasc Surg 2009;138:1363-9.

13. Pacini D, Leone A, Belotti LM, et al. Acute type A aortic dissection: significance of multiorgan malperfusion. Eur J Cardiothorac Surg 2013;43:820-6.

14. Beaulieu RJ, Arnaoutakis KD, Abularrage CJ, et al. Comparison of open and endovascular treatment of acute mesenteric ischemia. J Vasc Surg 2014;59:159-64.

15. Charlton-Ouw KM, Azizzadeh A, Sandhu HK, et al. Management of common carotid artery dissection due to extension from acute type A (DeBakey I) aortic dissection. J Vasc Surg 2013;58:910-6.

Cite this article as: Grimm JC, Magruder JT, Crawford TC, Sciortino CM, Zehr KJ, Mandal K, Conte JV, Cameron DE, Black JH, Price JE. Differential outcomes of type A dissection with malperfusion according to affected organ system. Ann Cardiothorac Surg 2016;5(3):202-208. doi: 10.21037/ acs.2016.03.11
16. Zieliński T, Wołkanin-Bartnik J, Janaszek-Sitkowska H, et al. Persistent dissection of carotid artery in patients operated on for type A acute aortic dissection--carotid ultrasound follow-up. Int J Cardiol 1999;70:133-9.

17. Tsukube T, Hayashi T, Kawahira T, et al. Neurological outcomes after immediate aortic repair for acute type A aortic dissection complicated by coma. Circulation 2011;124:S163-7.

18. Charlton-Ouw KM, Sritharan K, Leake SS, et al. Management of limb ischemia in acute proximal aortic dissection. J Vasc Surg 2013;57:1023-9. 